\title{
TU/e EN⿴HONE

\section{Simulation of mode-locking by nonlinear polarization rotation in a semiconductor optical amplifier}

\section{Citation for published version (APA):}

Li, Z., Yang, X., Tangdiongga, E., Ju, H., Khoe, G. D., Dorren, H. J. S., \& Lenstra, D. (2005). Simulation of mode-locking by nonlinear polarization rotation in a semiconductor optical amplifier. IEEE Journal of Quantum Electronics, 41(6), 808-816. https://doi.org/10.1109/JQE.2005.846689

DOI:

10.1109/JQE.2005.846689

Document status and date:

Published: 01/01/2005

\section{Document Version:}

Publisher's PDF, also known as Version of Record (includes final page, issue and volume numbers)

\section{Please check the document version of this publication:}

- A submitted manuscript is the version of the article upon submission and before peer-review. There can be important differences between the submitted version and the official published version of record. People interested in the research are advised to contact the author for the final version of the publication, or visit the $\mathrm{DOI}$ to the publisher's website.

- The final author version and the galley proof are versions of the publication after peer review.

- The final published version features the final layout of the paper including the volume, issue and page numbers.

Link to publication

\section{General rights}

Copyright and moral rights for the publications made accessible in the public portal are retained by the authors and/or other copyright owners and it is a condition of accessing publications that users recognise and abide by the legal requirements associated with these rights.

- Users may download and print one copy of any publication from the public portal for the purpose of private study or research.

- You may not further distribute the material or use it for any profit-making activity or commercial gain

- You may freely distribute the URL identifying the publication in the public portal.

If the publication is distributed under the terms of Article 25fa of the Dutch Copyright Act, indicated by the "Taverne" license above, please follow below link for the End User Agreement:

www.tue.nl/taverne

Take down policy

If you believe that this document breaches copyright please contact us at:

openaccess@tue.nl

providing details and we will investigate your claim. 


\title{
Simulation of Mode-Locking by Nonlinear Polarization Rotation in a Semiconductor Optical Amplifier
}

\author{
Z. Li, X. Yang, E. Tangdiongga, H. Ju, G.-D. Khoe, Fellow, IEEE, H. J. S. Dorren, and D. Lenstra
}

\begin{abstract}
We present a theoretical investigation of a mode locked laser that has a semiconductor optical amplifier (SOA) in its ring cavity. A mode-locked train of narrow pulses is obtained by combining nonlinear polarization rotation in the SOA and a polarization filter whose polarization axis is set such that the tail of optical pulses is removed in each cavity round-trip. The pulse narrowing process is demonstrated numerically and good qualitative agreement with experiments in our previous work is achieved. The pulse performance is largely determined by the ultrafast SOA gain dynamics and the cavity dispersion. Our simulation shows that the laser can produce a pulse train of subpicosecond pulsewidth at a repetition rate of $28 \mathrm{GHz}$ for a moderate SOA current level. We observe that the laser can switch itself on or off depending on the initial pulse.
\end{abstract}

Index Terms-Fiber ring laser, mode-locked ring laser, polarization switching, self-induced nonlinear polarization rotation, semiconductor optical amplifier (SOA).

\section{INTRODUCTION}

$\mathbf{L}$ IGHT sources capable of producing short optical pulses play a key role in optical communication systems [1]. Apart from applications in transmission systems, well-shaped optical pulses as short as several hundred femtoseconds may be needed for creating ultrashort switching windows in optical switches. Mode-locking is a widely used technique to generate short pulses [2]. Among the many available mode-locking schemes, nonlinear polarization rotation in optical fiber nonlinearities is often employed. Pulses as short as 42 fs have been generated by a passively mode-locked fiber ring laser based on nonlinear polarization rotation [3]. A clear disadvantage of employing fiber nonlinearities for mode-locking is the large

Manuscript received December 2, 2004; revised February 18, 2005. This work was supported by The Netherlands Organization for Scientific Research (NWO) through a National Research Council (NRC) Photonics grant, in part by the Technology Foundation STW under the the "Innovational Research Incentives Scheme Program," and in part by the Ministry of Economic Affairs under the technology program "Toward Freeband Communication Impulse."

Z. Li, E. Tangdiongga, H. Ju, G. D. Khoe, and H. J. S. Dorren are with the COBRA Research Institute, Eindhoven University of Technology, Eindhoven 5600 MB, The Netherlands (e-mail: zhonggui.li@tue.nl; E.Tangdiongga@tue.nl; h.ju@tue.nl; g.d.khoe@tue.nl; H.J.S.dorren@tue.nl).

X. Yang was with the COBRA Research Institute, Eindhoven University of Technology, Eindhoven 5600 MB, The Netherlands. He is now with the Photonics System Group, Department of Physics, University College Cork, Cork, Ireland (e-mail: x.yang@ucc.ie).

D. Lenstra is with the COBRA Research Institute, Eindhoven University of Technology, Eindhoven 5600 MB, The Netherlands and also with the Division of Physics and Astronomy, Vrije Universiteit, Amsterdam, The Netherlands (e-mail: d.lenstra@tue.nl).

Digital Object Identifier 10.1109/JQE.2005.846689 amount of pulse energies ( $\sim 50$ pJ for a 500-fs pulse) which are necessary in the optical fiber in order to utilize the weak nonlinearity in the optical fiber [3]. The long optical fiber cavity, as well as the high peak power of the optical pulses, limits the system to operate only at low repetition rates.

Nonlinear polarization rotation in a semiconductor optical amplifier (SOA) for optical signal processing has been investigated theoretically by [4]-[6], and experimentally in the context of wavelength conversion [7]-[9], all-optical switching [10]-[12], and an optical flip-flop memory [13]. These experiments were performed in the continuous-wave regime [9], [13] as well as in the pulsed regime [7], [8], [11]. Self-polarization rotation, i.e., nonlinear polarization rotation caused by the pulse itself, has also been utilized for all-optical signal processing [14]-[16].

Recently, we have demonstrated mode-locked pulses in a passively mode-locked ring laser, by using nonlinear self-polarization rotation in a SOA [17]. The pulses had duration of $800 \mathrm{fs}$. Although mode-locking using self-induced nonlinear polarization rotation in an SOA was already discussed in [18], to the best of our knowledge, the work in [17] was the first demonstration of this scheme. In [18], pulse narrowing and mode-locking due to self-polarization rotation has been investigated numerically. The pulse narrowing observed in their study was not counteracted by a broadening mechanism, such as group-velocity dispersion and ultrafast carrier dynamics. This gave the model in [18] only limited validity.

In this paper, using a consistent model with ultrafast gain dynamics [6], we will describe in detail how an initially broad pulse can be shortened using nonlinear polarization rotation in the SOA and what the condition is for the pulse to acquire enough round-trip gain to build up. The value of the linewidth enhancement factor of the SOA is found to be of crucial importance in the pulse built-up process. We will show simulations of the pulse evolution process, including the evolution of the spectrum. The final pulsewidth is found to be limited by ultrafast gain dynamics in the SOA combined with the dispersion effects in the laser cavity. We will also show that the highest possible repetition rate of the output pulses can be increased by increasing the injection current, but beyond a certain current unstable pulse patterns emerge.

\section{PRINCIPLE OF OPERATION}

The system setup is given in Fig. 1. The ring laser is composed of an SOA, followed by a polarization controller (PC), an optical isolator, an optical filter, an optical asymmetric output coupler, 


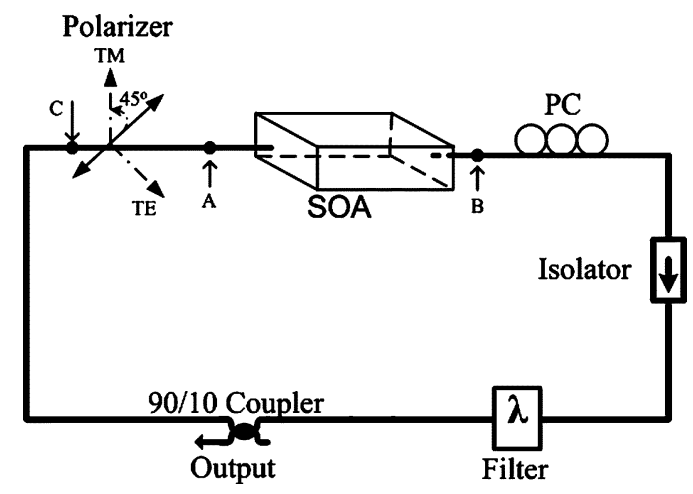

Fig. 1. System setup of the SOA-based fiber ring laser. SOA: semiconductor optical amplifier; PC: polarization controller. The linear polarizer has its transmitted polarization under $45^{\circ}$ with respect to the TE and TM axes of the SOA.

and a polarizer. The isolator is introduced in the cavity to keep the signal to propagate in one direction. The coupler is used to monitor the signal in the ring cavity. When the input optical intensity (point A in Fig. 1) is sufficiently low, the SOA operates in the linear regime. The polarization state at point $\mathrm{A}$ in Fig. 1 is linear and is set to $45^{\circ}$ to the transverse electric (TE) and transverese magnetic (TM) axes of the SOA. The two orthogonal polarization components in the amplifier collect different phases and gains. This causes intensity-independent polarization conversion at the SOA output. Note, in passing by, that in this context one often uses the word polarization rotation. However, this may not be a correct description because in general the TE and TM components will collect different phase shifts while propagating. Therefore an initial linear polarization state will not only rotate, but in general also assume a certain degree of ellipticity.

Now suppose the input optical intensity becomes high enough to saturate the amplifier. Then, TE and TM component collect different intensity-dependent phases and amplitudes. This implies that different parts of the output pulse assume different polarization states and this property makes it possible to cut away the pulse part that has the same polarization as in the low input intensity case. To realize this, one uses a combination of the PC and the polarizer, which are adjusted properly to achieve the required functionality: the $\mathrm{PC}$ is adjusted in such a way that for small signal case the polarization of the pulse (point $C$ in Fig. 1) is orthogonal to the axis of the polarizer, while the latter has been oriented at $45^{\circ}$ to the TE and TM axes of the SOA. By doing so, a low-intensity input signal to the SOA will be removed from the ring, preventing the signal from building up. On the other hand, for a sufficiently strong input pulse, the self-induced nonlinear polarization rotation of the high-intensity part of the pulse will create a nonzero but shortened pulse behind the polarizer. If the SOA has enough gain, a net round-trip gain for pulses can be established. In fact, the nonlinear polarization rotation, combined with the PC and the polarizer, has the same functionality as a saturable absorber. This provides the basic mechanism for our mode-locking system.

In agreement with the above description, we found that the system is by itself bistable in the sense that either an output train of short strong pulses or no output at all occurs in the system depending on the initial conditions. Therefore, this mode-locked ring laser could act as a basic element for a flip-flop memory system, and may find its application in optical signal processing systems.

In the experiment in [17], one usually starts in the nonoptimized setting with a quasi-continuous oscillation in the laser. Then during the adjustment of PC, the system is disturbed to generate optical pulses randomly in the cavity. Some pulses happen to satisfy the conditions that are set by the PC and the polarizer such that they can pass through the polarizer and reach the SOA again after one round-trip in the cavity. After each round-trip those pulses become narrower and converge toward a stable pulse train.

\section{MODEL}

The nonlinear optical pulse propagation in the SOA is simulated using the model developed in [6]. This model is based on the decomposition of the polarized optical field into TE and TM components that interact via the gain saturation. The extensive description given in [6] will not be repeated here. It accounts for the influences of two-photon absorption, free-carrier absorption, self- and cross-phase modulation, carrier heating, and spectral and spatial hole burning. SOAs with unstrained bulk active material have much larger TE amplification than TM, which is due to the different confinement factors. Therefore, the SOAs active material is often strained in order to enhance the gain of TM with respect to TE, making the SOA less polarization dependent. In our model the strain-induced gain anisotropy is taken into account by introducing the separate hole populations for TE and TM and corresponding valence subband structures for the TE and TM transitions [19]. According to the pulse phase evolution equations given in [6], TE and TM components experience exactly the same phase due to two-photon absorption. Within that approximation two-photon absorption has no direct influence on the evolution of the pulse polarization.

The PC and polarizer are modeled according to [20]. By representing the electrical field as the Jones vector, the functions of the PC and the polarizer can be written in $2 \times 2$ matrices which act on the electrical field vector.

Suppose at point A in Fig. 1, a weak electric field is present with normalized polarization vector given by

$$
\frac{1}{\sqrt{2}}\left[\begin{array}{l}
1 \\
1
\end{array}\right] \text {. }
$$

Equation (1) represents linearly polarized light under $45^{\circ}$ with respect to the TE and TM axes of the SOA. After propagation through the SOA, the TE and TM components acquire different amplitudes and phases. Hence, we can write the field at B as

$$
\frac{1}{\sqrt{2}}\left[\begin{array}{c}
\tau_{0}^{\mathrm{TE}} e^{i \phi_{0}^{\mathrm{TE}}} \\
\tau_{0}^{\mathrm{TM}} e^{i \phi_{0}^{\mathrm{TM}}}
\end{array}\right]
$$

where $\tau_{0}^{\mathrm{TE}}$ and $\tau_{0}^{\mathrm{TM}}$ are the linear amplifications and $\phi_{0}^{\mathrm{TE}}, \phi_{0}^{\mathrm{TM}}$ the linear phase shifts. Now, the PC is adjusted in such a way that the polarization state at $\mathrm{C}$ is orthogonal to that at $\mathrm{A}$

$$
\frac{1}{\sqrt{2}} \beta\left[\begin{array}{c}
1 \\
-1
\end{array}\right]
$$


where by assuming lossless propagation from $\mathrm{B}$ to $\mathrm{C}, \beta$ can be written as

$$
\beta=\frac{1}{\sqrt{2}} \sqrt{\left(\tau_{0}^{\mathrm{TE}}\right)^{2}+\left(\tau_{0}^{\mathrm{TM}}\right)^{2}} .
$$

The unitary matrix $U$, representing the $\mathrm{PC}$, can be written as

$$
\begin{aligned}
U & =\frac{1}{\sqrt{2\left(\left(\tau_{0}^{\mathrm{TE}}\right)^{2}+\left(\tau_{0}^{\mathrm{TM}}\right)^{2}\right)}} \\
& \times\left[\begin{array}{ll}
\left(\tau_{0}^{\mathrm{TE}}+\tau_{0}^{\mathrm{TM}}\right) e^{-i \phi_{0}^{\mathrm{TE}}} & \left(\tau_{0}^{\mathrm{TM}}-\tau_{0}^{\mathrm{TE}}\right) e^{-i \phi_{0}^{\mathrm{TM}}} \\
\left(\tau_{0}^{\mathrm{TM}}-\tau_{0}^{\mathrm{TE}}\right) e^{-i \phi_{0}^{\mathrm{TE}}} & -\left(\tau_{0}^{\mathrm{TE}}+\tau_{0}^{\mathrm{TM}}\right) e^{-i \phi_{0}^{\mathrm{TM}}}
\end{array}\right]
\end{aligned}
$$

which can be easily checked from the requirement

$$
U\left[\begin{array}{c}
\tau_{0}^{\mathrm{TE}} e^{i \phi_{0}^{\mathrm{TE}}} \\
\tau_{0}^{\mathrm{TM}} e^{i \phi_{0}^{\mathrm{TM}}}
\end{array}\right]=\frac{1}{\sqrt{2}} \sqrt{\left(\tau_{0}^{\mathrm{TE}}\right)^{2}+\left(\tau_{0}^{\mathrm{TM}}\right)^{2}}\left[\begin{array}{c}
1 \\
-1
\end{array}\right] .
$$

It should be noted in (5) that $U$ is calculated from simulations in the linear amplification regime. The polarizer is adjusted such that the field component along $(1,1)$ will be passed through and that along $(1,-1)$ will be blocked.

Now suppose that the input intensity to the SOA increases to values that are large enough to induce polarization-dependent nonlinear gain saturation. Then, after the PC some field component will be generated along $(1,1)$, which can be expressed as

$$
\frac{1}{2}\left[\begin{array}{ll}
1 & 1 \\
1 & 1
\end{array}\right] U\left[\begin{array}{l}
\gamma^{\mathrm{TE}} \\
\gamma^{\mathrm{TM}}
\end{array}\right]
$$

where $\left(\gamma^{\mathrm{TE}}, \gamma^{\mathrm{TM}}\right)$ represents the field at B. The field, represented by (7), will enter the SOA again.

A spectral filter is included in the simulations in order to account for dispersion effects in the experiment. This filter also accounts partly for the gain dispersion. The filter in the system is modeled as a Lorentzian filter with a full-width at half-maximum (FWHM) of $2 D$. The transfer function of the filter is

$$
f(\omega)=\frac{D}{D-i\left(\omega-\omega_{0}\right)}
$$

where $\omega_{0}$ is the central angular frequency of the filter. The filter is implemented in the frequency domain using a fast Fourier transform (FFT) algorithm while an eventual filter-induced change of the pulse polarization is included in the unitary matrix $U$.

\section{Simulation Results AND Discussion}

The SOA has a strained bulk active region of $250 \mu \mathrm{m}$ and active volume of $50 \mu \mathrm{m}^{3}$ [6]. Other parameters are listed in Table I. All calculations were performed for $160-\mathrm{mA}$ injection current except stated otherwise. The parameter values of the SOA were chosen in such a way that stable mode locking occurs. This is
TABLE I

SOA PARAMETER DEFINITIONS AND THEIR VALUES

\begin{tabular}{lll}
\hline Parameter & Value & units \\
\hline Active volume & $250 \times 2 \times 0.1$ & $\mu \mathrm{m}^{3}$ \\
Confinement factor TE/TM/TPA & $0.18 / 0.13 / 0.5$ & \\
Linewidth enhancement factor TE/TM/TPA & $4 / 4 /-1$ & \\
FCA coefficient in conduction band & $3 \times 10^{-9}$ & ${\mu \mathrm{m}^{2}}^{2}$ \\
FCA coefficient in valence band & 0 & $\mu \mathrm{m}^{2}$ \\
Electron-hole pair lifetime & 300 & $\mathrm{ps}$ \\
Gain coefficient TE/TM & $1.4 \times 10^{-5}$ & $\mu \mathrm{m}^{3} / \mathrm{ps}$ \\
Group velocity & 100 & $\mu \mathrm{m} / \mathrm{ps}$ \\
Internal loss & 0.00175 & $\mu \mathrm{m}^{-1}$ \\
Optical transition energies(conduction band) & $0.05,1.12$ & $\mathrm{eV}$ \\
Optical transition energies(valence band) & $0.005,0.11$ & $\mathrm{eV}$ \\
Carrier-carrier scattering time in conduction band & 0.1 & $\mathrm{ps}$ \\
Carrier-carrier scattering time in valence band & 0.05 & $\mathrm{ps}$ \\
Carrier-phonon relaxation time in conduction band & 0.7 & $\mathrm{ps}$ \\
Carrier-phonon relaxation time in valence band & 0.25 & $\mathrm{ps}$ \\
TPA coefficient & $2 \times 10^{-9}$ & $\mu \mathrm{m}^{2}$ \\
Optical transition state density & $3.6 \times 10^{6}$ & $\mu \mathrm{m}^{-3}$ \\
\hline TPA: TwO photon absorption & &
\end{tabular}

TPA: Two photon absorption

FCA: Free carrier absorption

not an easy task because the region in the parameter space that yields stable mode-locking appears to be quite small. For these values, the small-signal gain for TE and TM components are 16.8 and $14.8 \mathrm{~dB}$, respectively.

Since the polarization rotation necessary for pulse narrowing is mainly due to nonlinear phase shifts, it is obvious that the linewidth enhancement factor $\alpha$ plays an important role. In our simulation, we use $\alpha=\alpha^{\mathrm{TE}}=\alpha^{\mathrm{TM}}$. The net pulse round-trip gain will increase with higher values of $\alpha$. Therefore, for a given injection current, $\alpha$ should be larger than some threshold value to cause the ring to build up optical pulse and to converge to a stable pulse train. The threshold is determined by the SOA parameters and the round-trip losses. If $\alpha$ is smaller than the threshold value, the pulse will be attenuated, irrespective of the intensity value of the initial pulse. This follows from the computation of the round-trip gain, which is taken as the ratio between the current optical intensity at point A in Fig. 1 and its previous intensity, shown in (9) at the bottom of the page, where $\tau_{0}^{\mathrm{TE}}, \tau_{0}^{\mathrm{TM}}, \varphi_{0}^{\mathrm{TE}}$, and $\varphi_{0}^{\mathrm{TM}}$ are the single-pass transmission and the phase of the pulse for TE and TM components in the low-input intensity case while $\tau^{\mathrm{TE}}, \tau^{\mathrm{TM}}, \varphi^{\mathrm{TE}}$, and $\varphi^{\mathrm{TM}}$ are the time-dependent single-pass transmission and phase of the pulse for TE and TM components in the nonlinear regime at point B in Fig. 1. The $t$-dependence in (9) originates from the fact that different parts of the pulse have different phase and intensity. The round-trip gain should be larger than $0 \mathrm{~dB}$ for the pulse to build up.

In Fig. 2, the gain at the pulse peak in the first round-trip is shown as a function of the initial pulse energy for different $\alpha$ for the injection current of $160 \mathrm{~mA}$. Throughout the paper "initial pulse" means the pulse in the zeroth round-trip, referring to the

$$
G(t)=\frac{\left(\tau_{0}^{\mathrm{TM}} \tau^{\mathrm{TE}}(t)\right)^{2}+\left(\tau_{0}^{\mathrm{TE}} \tau^{\mathrm{TM}}(t)\right)^{2}-2 \tau_{0}^{\mathrm{TE}} \tau_{0}^{\mathrm{TM}} \tau^{\mathrm{TE}}(t) \tau^{\mathrm{TM}}(t) \cos \left\{\left(\varphi^{\mathrm{TM}}(t)-\varphi^{\mathrm{TE}}(t)\right)-\left(\varphi_{0}^{\mathrm{TM}}-\varphi_{0}^{\mathrm{TE}}\right)\right\}}{2\left(\left(\tau_{0}^{\mathrm{TE}}\right)^{2}+\left(\tau_{0}^{\mathrm{TM}}\right)^{2}\right)}
$$




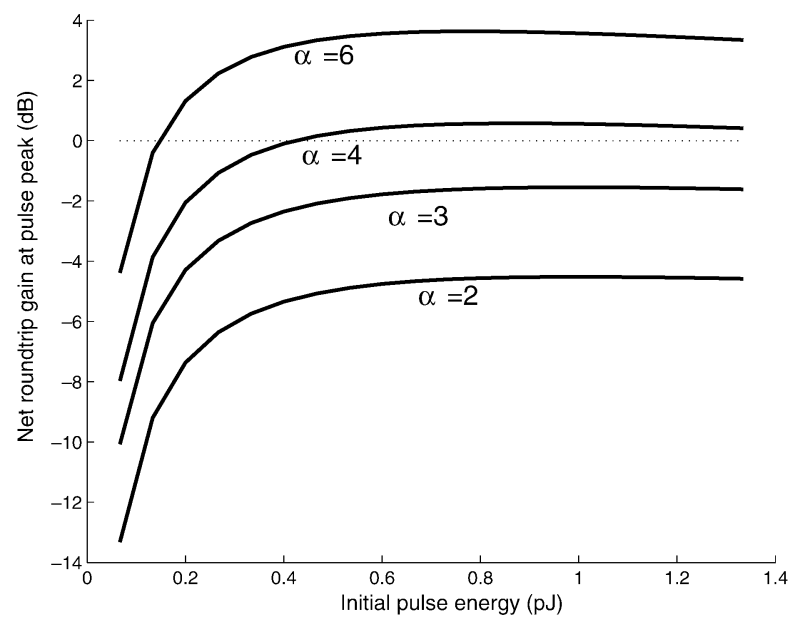

Fig. 2. Round-trip gain at the pulse peak versus the initial pulse energy for different linewidth enhancement factors $\alpha$ in the first round-trip. (Initial pulse shape is chirp-free Gaussian with 2-ps rms width).

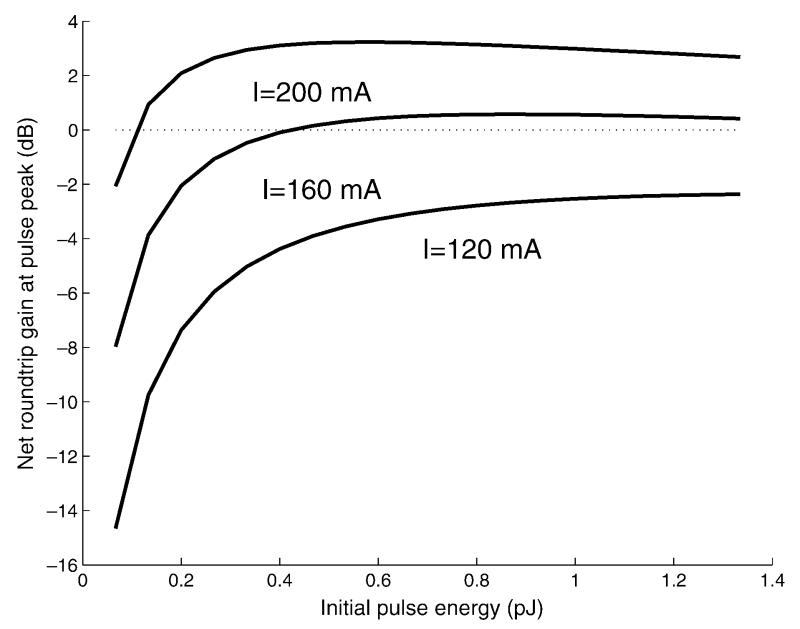

Fig. 3. Net round-trip gain at the pulse peak versus the initial pulse energy for different injection current in the first round-trip. (Linewidth enhancement factor $\alpha$ is 4 , other parameters as in Fig. 2).

initial values set in the computer code. One readily observes that the linewidth enhancement factor $\alpha$ plays a crucial role in the pulse built-up process. For the pulse to be amplified and narrowed, $\alpha$ should be larger than 4 . For a given $\alpha$, the loop gain can be increased by pumping larger current into the active region. That is shown in Fig. 3 for $\alpha=4$. However, for very small $\alpha$ it may become impossible to obtain $G>0 \mathrm{~dB}$ because of gain saturation at higher currents. In Figs. 2 and 3, initial pulses were taken to be Gaussian pulse with a root mean square (rms) width of 2 ps.

The simulations show that initial pulses with insufficient intensity cannot induce large enough phase shifts between TE and TM components for the pulse to acquire positive round-trip gain. This is shown in Fig. 4, where a 2-ps 0.22-pJ Gaussian initial pulse is seen to decrease after each round-trip. When the initial pulse intensity is increased beyond a threshold value, the pulse is amplified and narrowed in every loop. After several tens of round-trips the pulse has evolved into a stable shape. This is shown in Fig. 5 for an unchirped Gaussian shaped initial pulse of

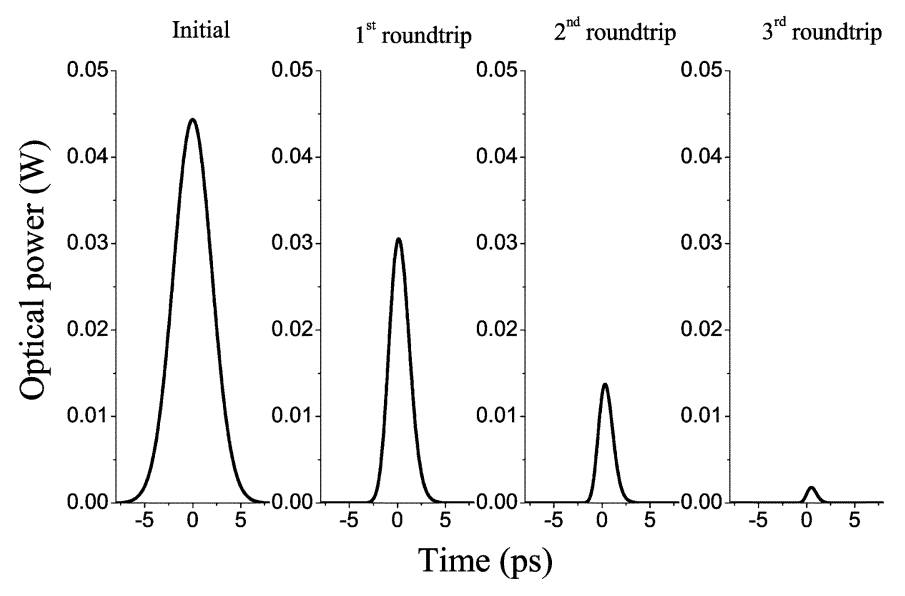

Fig. 4. Initial pulse has insufficient energy for a net positive round-trip gain, even though the parameter values are within the mode-locking regime. Therefore, the pulse is attenuated after each round-trip. Note that narrowing takes place. The initial Gaussian pulse has a width of $2 \mathrm{ps}$, is unchirped and has energy of $0.22 \mathrm{pJ}$. After a few round-trips, the pulse has disappeared. The injection current is $160 \mathrm{~mA}$ and the linewidth enhancement factor is 4 .

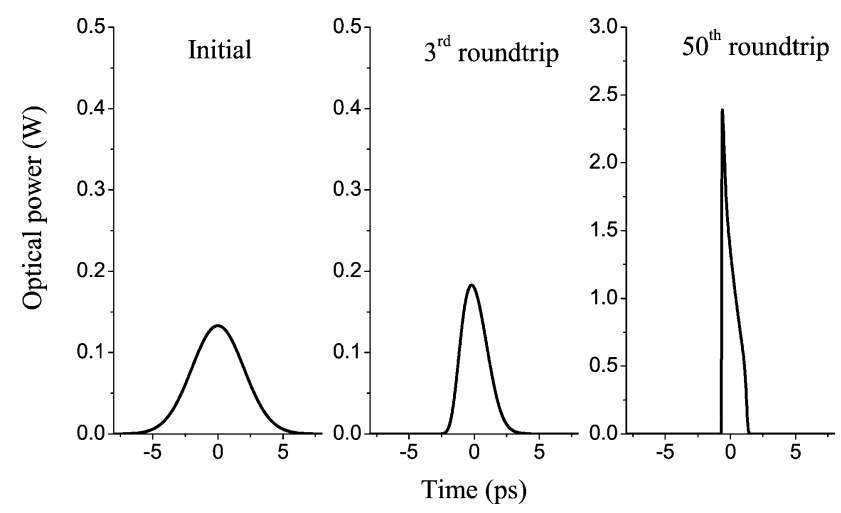

Fig. 5. Numerical simulation of the pulse built-up for the same laser as in Fig. 4. Initially the pulse has duration of 2 ps and its energy is $0.66 \mathrm{pJ}$. After 3 round-trips, the pulse duration has decreased to $815 \mathrm{fs}$ and the pulse energy has decreased to $0.45 \mathrm{pJ}$. After 25 round-trips, the pulse has been shorted further to the ultimate width of $537 \mathrm{fs}$ and energy $2.33 \mathrm{pJ}$. The filter bandwidth is $3.2 \mathrm{THz}$. The injection current is $160 \mathrm{~mA}$ and the linewidth enhancement factor is 4 .

2-ps duration and 0.66-pJ energy. A filter of $3.2 \mathrm{THz}$ (FWHM) was used. The dispersion in the laser cavity, simulated by the filter, is estimated to be $0.235 \mathrm{ps} / \mathrm{nm}$. One can observe in Fig. 5 that after about 25 round-trips the stable state is achieved, which is characterized by $537-\mathrm{fs}$ (rms) duration output pulse train. The energy of each pulse is $2.33 \mathrm{pJ}$.

The evolutions of the pulse peak power and the pulse rms width are presented in Fig. 6, in which the solid line corresponds to the above mentioned case of Fig. 5 and the dotted line corresponds to an initial pulse of $4 \mathrm{ps}$, unchirped Gaussian pulse with the energy of 1.33 pJ. Two other cases are also shown in Fig. 6, i.e., the dot-dashed line, which corresponds to a super Gaussian pulse [21] (order $m=3$ ) with white intensity noise, and the dashed line, which corresponds to a pulse with non-Gaussian shape. Fig. 6 illustrates that the system with different initial pulses evolves into the same final state, i.e., a stable identical pulse train. In Fig. 7, the pulse evolution is shown for the case of the noisy super-Gaussian initial pulse. 


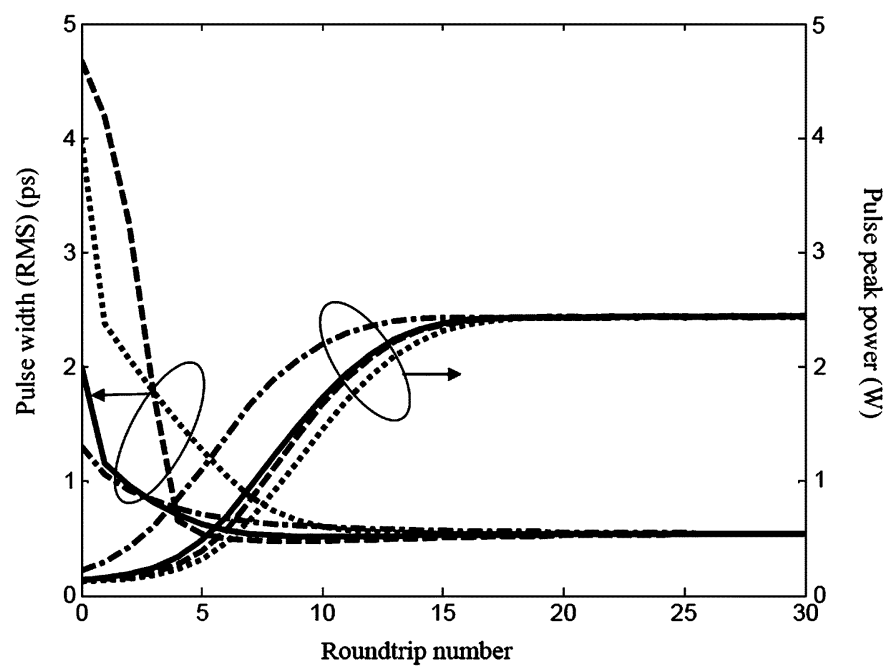

Fig. 6. Evolution of the width and the peak power in the pulse built-up process. The solid line corresponds to a 2-ps Gaussian initial pulse; the dot-dashed line corresponds to a super-Gaussian pulse (order $m=3$ ) with white intensity noise; the dotted line corresponds to a 4-ps Gaussian initial pulse, and the dashed line corresponds to an arbitrary non-Gaussian pulse. The peak power in all the cases is the same, except for the super-Gaussian case due to the contribution of noise. The filter bandwidth is $3.2 \mathrm{THz}$.

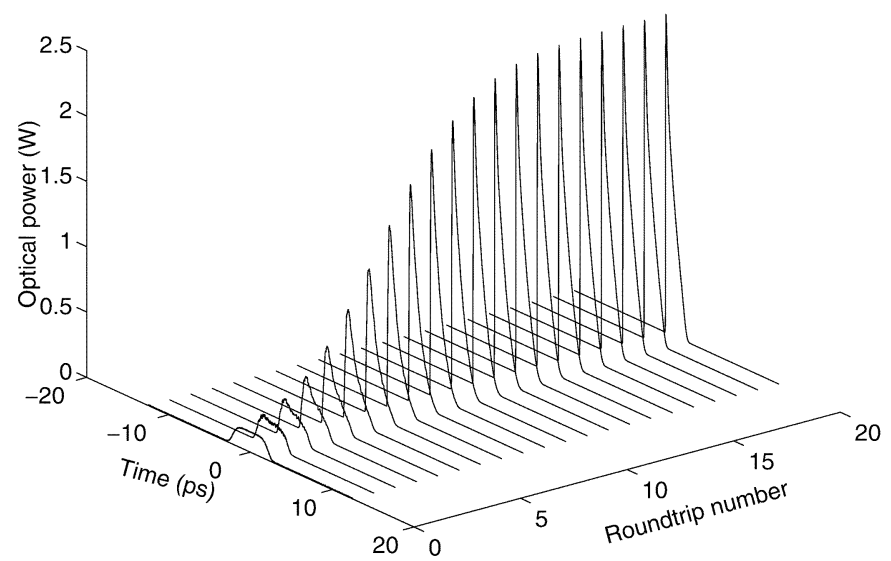

Fig. 7. Pulse evolution in the 20 round-trips during the built-up process for a super Gaussian pulse (order $m=3$ ) with white intensity noise. The initial pulse energy is $0.54 \mathrm{pJ}$ and the pulsewidth is $1.27 \mathrm{ps}$. The filter bandwidth is $3.2 \mathrm{THz}$.

In Fig. 8, the optical spectra corresponding to the pulses shown in Fig. 5 are presented. The spectrum steadily broadens after each round-trip and finally the pulses achieve their stable spectrum. The initial Gaussian pulse is unchirped, and its product of the rms pulse temporal width and the rms spectral width is $\Delta t \Delta v \approx 0.08$ [22]. The stable output pulse has a rms temporal width of 537 fs and a rms frequency width of $0.56 \mathrm{THz}$, hence, their time-bandwidth product $\Delta t \Delta v=0.3$. The relatively large value is mainly caused by the pulse shape in which the leading edge is much steeper than the trailing edge. By comparing this value with the time-bandwidth product of the same pulse but without chirp, which is 0.19 , we conclude that the output pulse is moderately chirped. It is also seen from Fig. 8 that the main frequency components of the pulse are red-shifted.

According to the system principle, the polarizer blocks the pedestal in the leading edge of the pulse and this causes the

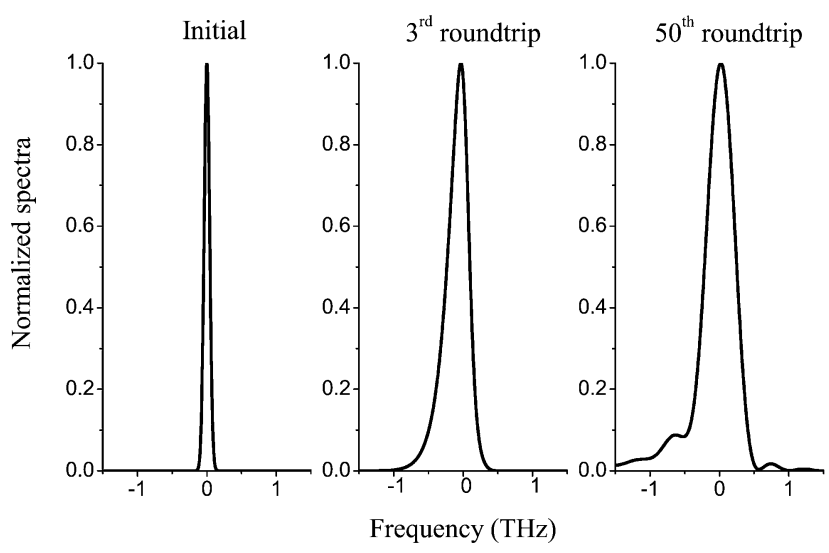

Fig. 8. Optical spectra corresponding to Fig. 5. Initially the spectral width is $40 \mathrm{GHz}$. After five round-trips, the spectral width has increased to $0.18 \mathrm{THz}$, and after 25 round-trips, the spectral width has stabilized at $0.56 \mathrm{THz}$. The origin in frequency axis is the initial pulse center frequency.

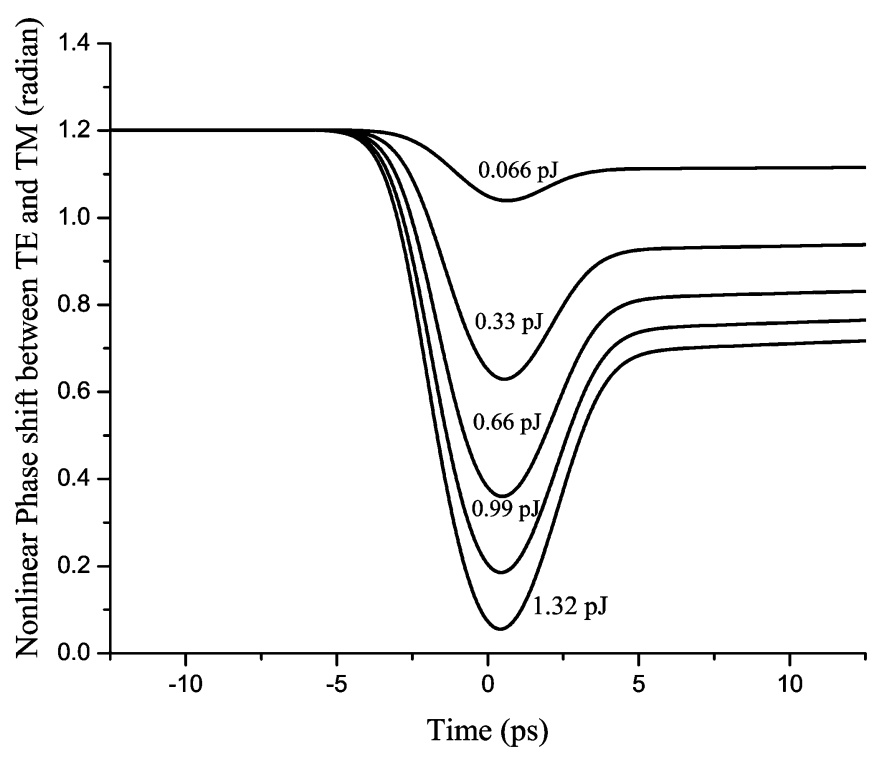

Fig. 9. Time-dependent nonlinear phase difference between the TE and TM components after the first round-trip, where the initial pulse is a 2-ps unchirped Gaussian pulse with different initial pulse energies, as indicated.

leading edge to be very steep (see Fig. 5). On the other hand, the trailing edge of the output pulse has a long tail, which is caused by the incomplete recovery of SOA gain and phase difference between TE and TM components. This is shown in Fig. 9, where the phase difference between the TE and TM components in one pulse after one round-trip for different initial pulse energies is presented. The corresponding asymmetry of the pulse shape is the reason why we choose rms width as a measure for the pulsewidth instead of FWHM, similarly for the pulse spectrum. We note here that the nonlinear phase difference between TE and TM components evolves in a similar way as the corresponding gains, which explains the importance of gain dynamics in the pulse evolution process. In Fig. 10, the polarization states are shown during the first, third, and 50th round-trips, in terms of azimuth and ellipticity angles. The intensity-dependent polarization states within the pulse duration can be clearly seen. We also observe from Fig. 10 the large residual effect in the trailing edge of the final output pulse. 


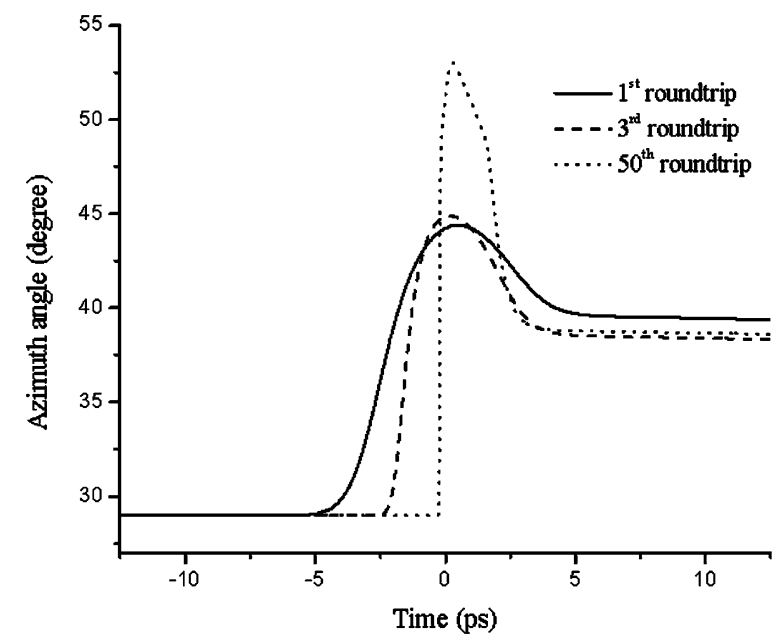

(a)

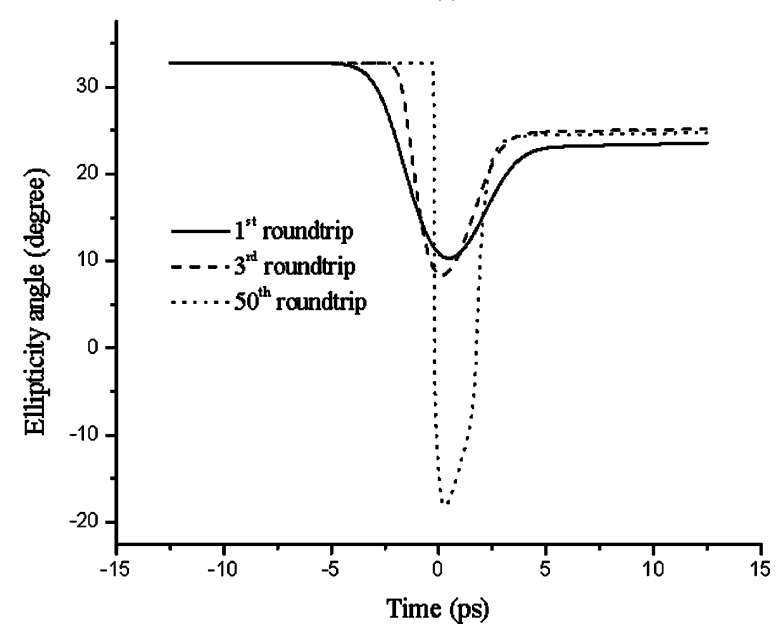

(b)

Fig. 10. (a) Azimuth angle and (b) ellipticity of the pulse at the exit of the SOA after various round-trips as indicated. The initial pulse is a 2-ps, unchirped Gaussian pulse with 0.66-pJ energy.

The output pulsewidth was found to depend on the width of the filter that is added in the model in order to account for the effect of dispersive effects. The stable pulsewidth decreased for the increased filter bandwidth, as expected. The question arises as to what determines the ultimate pulsewidth. As shown in Table II, the pulsewidth decreased to 350 fs when the carrierphonon scattering time in the conduction band was decreased to $500 \mathrm{fs}$. The pulsewidth increased to $645 \mathrm{fs}$ when the carrierphonon scattering time was increased to $1 \mathrm{ps}$. The pulsewidth is also found to be injection current dependent. As shown in Fig. 11, the final pulsewidth increases from 0.15 to 0.82 ps when the injection current is increased from 152 to $175 \mathrm{~mA}$. In the simulations, the carrier phonon relaxation time is kept as $700 \mathrm{fs}$ and the FWHM of the filter bandwidth is $3.2 \mathrm{THz}$. When the injection current is below $152 \mathrm{~mA}$, the system cannot build up pulses anymore because the net round-trip gain falls below $0 \mathrm{~dB}$. On the other hand, when the injection current is further increased to higher than $180 \mathrm{~mA}$, instabilities instead of mode-locking occur. To understand this current dependence, one should realize that the long-lived tail in Fig. 9 (or Fig. 10) also depends upon the current. In fact, for high currents the
TABLE II

Pulse rms Width Versus CARRIER PhONON RElaXATION Time AT 160 mA

\begin{tabular}{cccc}
\hline Carrier-phonon relaxation time (ps) & 0.5 & 0.7 & 1 \\
\hline Final pulse RMS width (ps) & 0.35 & 0.455 & 0.645 \\
\hline
\end{tabular}

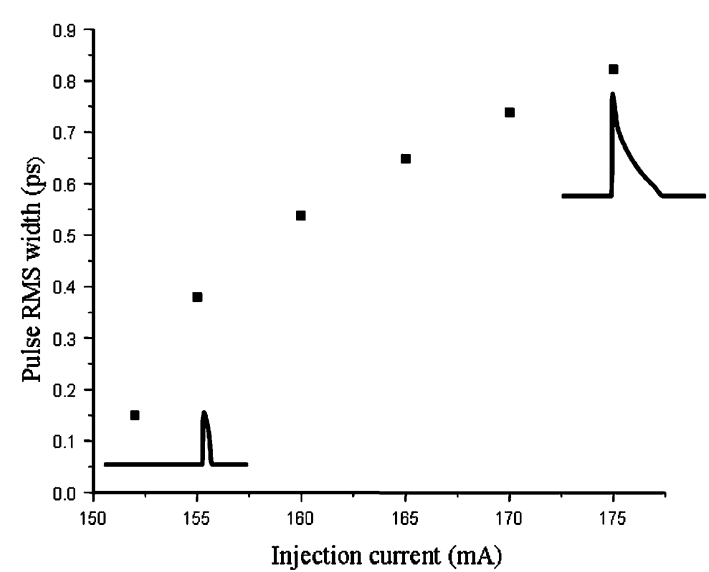

Fig. 11. Stable pulse rms width under different injection currents. The carrier-phonon relaxation time is $700 \mathrm{fs}$. The filter bandwidth is $3.2 \mathrm{THz}$.

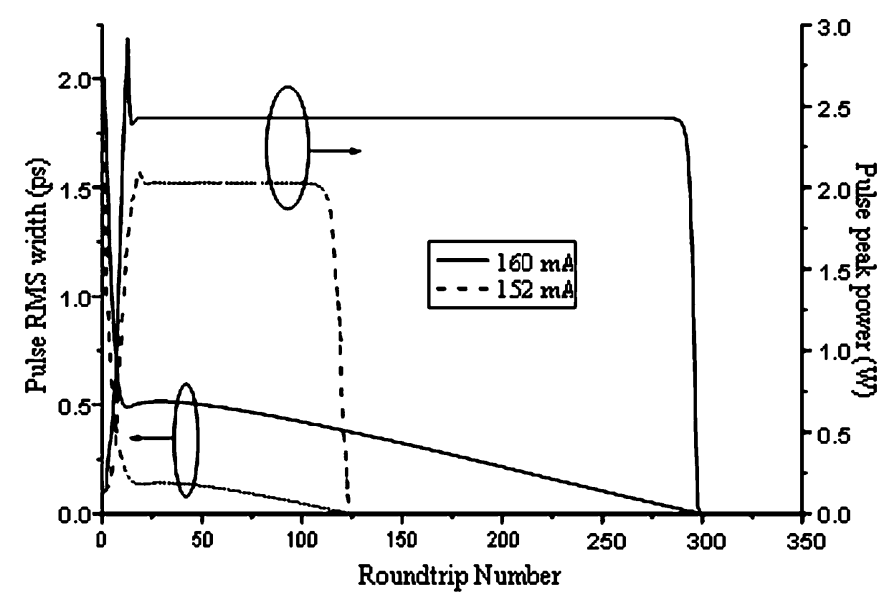

Fig. 12. Pulse rms width evolution with time when there is no filter in the cavity under different currents (160 and $152 \mathrm{~mA}$ ). Initially, the pulse has duration of $2 \mathrm{ps}$ and its energy is $0.66 \mathrm{pJ}$.

long-lived tail in the nonlinear phase shifts between TE and TM components is further away from the initial value than for low currents. This makes the pulse compression for the high currents less efficient than for the low currents.

When the filter is removed from the cavity (corresponding to the ideal dispersion-free operation), the pulsewidth decreases continuously in time, as shown in Fig. 12. Here we observe a rapid initial drop in pulsewidth, followed by a much more gradual and ultimately even linear decrease to zero. In the first 20 round-trips, the pulse peak power evolves in the same way as described in Fig. 6 while the pulse energy increases to its maximum value after its initial drop in the first several round-trips. After about 20 round-trips, the pulse peak power remains approximately constant and decreases dramatically after 110 round-trips for $152 \mathrm{~mA}$ and 280 round-trips for $160 \mathrm{~mA}$. The pulsewidth decreased so much that the pulse energy cannot maintain the required gain saturation anymore: the pulses fade away. Note that all these behaviors refer to a situation without 
TABLE III

(a) ACHiEVABle Bit Rate Versus CARrier LifETime at $160 \mathrm{~mA}$ (b) Achievable Bit Rate Versus InJeCtion CurRent (CARRIER LIFETIME IS $300 \mathrm{ps)}$

\begin{tabular}{cccc}
\hline Carrier lifetime (ps) & 200 & 300 & 500 \\
\hline Achievable bit rate @ $160 \mathrm{~mA}(\mathrm{GHz})$ & 20 & 5 & 2.5 \\
\hline
\end{tabular}

(a)

\begin{tabular}{cccc}
\hline Injection current $(\mathrm{mA})$ & 160 & 170 & 200 \\
\hline Achievable bit rate $(\mathrm{GHz})$ & 5 & 8 & 28 \\
\hline
\end{tabular}

(b)

any dispersion. Clearly, the effect of dispersion is to stabilize the pulsewidth as is shown in Fig. 6. Looking again at Fig. 12, the values of the pulsewidth directly after the initial drops are close to the corresponding values in Fig. 11. Hence, the current dependence in Fig. 12 is fully consistent with Fig. 11.

The pulse repetition rate in the experiments was $14 \mathrm{MHz}$ [16]. In our simulations so far, the SOA fully recovered during one round-trip, i.e., the cavity length was assumed to be long enough for such recovery, leading to upper bounds on the repetition rate. We also investigated the effect of partial SOA recovery during one round-trip. In a series of simulation experiments we found that the achievable pulse repetition rate is constrained by the carrier recovery time. If the repetition rate increases, the intensity of the pulses decreases due to gain depletion, until the pulse round-trip gain falls below $0 \mathrm{~dB}$ and the laser switches off. Once switched off, the system cannot start mode-locking again due to the bistable behavior. As presented in Table III(a), the pulse repetition rate is limited up to $5 \mathrm{GHz}$ when the carrier lifetime is assumed to be $300 \mathrm{ps}$. The pulse repetition rate is increased to $20 \mathrm{GHz}$ when the carrier lifetime is assumed to be $200 \mathrm{ps}$. The pulse repetition rate decreased to about $2.5 \mathrm{GHz}$ when the carrier lifetime is assumed to be $500 \mathrm{ps}$. At the same time, the pulse repetition rate can be improved by injecting larger current. In Table III(b), the achievable repetition rates at different injection currents are presented. As clearly seen, the repetition rate can be increased when the injection current is higher. However, after a critical current, $200 \mathrm{~mA}$ in our case, instabilities instead of mode-locking occur.

\section{SUMMARY AND CONCLUSION}

In conclusion, the pulse narrowing process in a passive modelocking system based on nonlinear polarization rotation in a SOA was modeled and simulated. The SOA model is based on the fact that TE and TM modes interact with each other via carriers. The model includes two-photon absorption, free-carrier absorption, self- and cross- phase modulation, carrier heating, and spectral hole burning. The PC and the polarizer were modeled as transmission matrices, which is an efficient way to model polarization-dependent effects.

We presented and discussed simulation results for the pulse built-up and narrowing as well as the pulse decay, depending on whether or not the initial pulse intensity is sufficiently high. The pulse narrowing mechanism is the self-induced nonlinear phase difference change between the TE and TM field components, which was found to be more than 1 radian when the input pulse energy is $1 \mathrm{pJ}$ (2-ps Gaussian pulse). The pulse built-up condition was investigated. By increasing the linewidth enhancement factor or the injection current, the required energy for the initial pulse to acquire net round-trip gain is decreased, which indicates that it is easier for the system to evolve into a stable mode-locking state. In the unrealistic case of dispersion-free operation, there is no fundamental limit to the pulse narrowing. However, in practice, the achievable pulsewidth is limited by both the cavity dispersion and the ultrafast gain dynamics, which is current dependent. For currents beyond a critical value, instabilities destroy the mode locking. The highest possible pulse repetition rate is increased by increasing the injection current while finally limited by the carrier lifetime. We obtained highest possible repetition rate of $28 \mathrm{GHz}$ at $200 \mathrm{~mA}$. In the experiment of [17], the pulsewidth (FWHM) and the spectral width (FWHM) are measured to be $800 \mathrm{fs}$ and $0.62 \mathrm{THz}$, respectively. In our simulation of the experiment, those values are $960 \mathrm{fs}$ and $0.53 \mathrm{THz}$, respectively. In terms of time-bandwidth product, the simulation value $(0.51)$ is close to the experiment value (0.49). Therefore, we conclude that the simulation is in agreement with the experiment.

\section{REFERENCES}

[1] M. Nakazawa, T. Yamamoto, and K. Tamura, "1.28 Tbit/s-70 km OTDM transmission using third- and fourth-order simultaneous dispersion compensation with a phase modulator," Electron. Lett., vol. 36, pp. 2027-2029, 2000.

[2] H. A. Haus, "Mode-locking of lasers," IEEE J. Sel. Topics Quantum Electron., vol. 6, pp. 1173-1185, Nov./Dec. 2000.

[3] L. E. Nelson, D. J. Jones, K. Tamura, H. A. Haus, and E. P. Ippen, "Ultrashort-pulse fiber ring lasers," Appl. Phys. B, vol. 65, pp. 277-294, 1997.

[4] Y. Takahashi, A. Neogi, and H. Kawaguchi, "Polarization dependent nonlinear gain in semiconductor optical amplifiers," IEEE J. Quantum Electron., vol. 34, no. 9, pp. 1660-1672, Sep. 1998.

[5] H. J. S. Dorren, D. Lenstra, Y. Liu, M. T. Hill, and G. D. Khoe, "Nonlinear polarization rotation in semiconductor optical amplifiers: Theory and application to all-optical flip-flop memories," IEEE J. Quantum Electron., vol. 39, no. 4, pp. 141-147, Apr. 2003.

[6] X. Yang, D. Lenstra, G. D. Khoe, and H. J. S. Dorren, "Nonlinear polarization rotation induced by ultrashort optical pulses in a semiconductor optical amplifier," Opt. Commun., vol. 223, pp. 169-179, 2003.

[7] M. F. C. Stephens, M. Asghari, R. V. Penty, and I. H. White, "Demonstration of ultrafast all-optical wavelength conversion utilizing birefringence in semiconductor optical amplifiers," IEEE Photon. Technol. Lett., vol. 9, no. 4, pp. 449-451, Jan. 1997.

[8] A. K. Mishra, X. Yang, D. Lenstra, G. D. Khoe, and H. J. S. Dorren, "All-optical wavelength conversion using nonlinear polarization rotation driven by $120 \mathrm{fs}$ optical pulses in a semiconductor optical amplifier," IEEE J. Sel. Top. Quantum Electron, vol. 10, no. 5, pp. 1180-1186, Sep./Oct. 2004.

[9] Y. Liu, M. T. Hill, E. Tangdiongga, H. de Waardt, N. Calabretta, G. D. Khoe, and H. J. S. Dorren, "Wavelength converter using nonlinear polarization rotation in a single semiconductor optical amplifier," IEEE Photon. Technol. Lett., vol. 15, no. 1, pp. 90-93, Jan. 2003.

[10] J. T. Hyland, G. T. Kennedy, A. Miller, and C. C. Button, "Picosecond all optical polarization switching in InGaAsP MQWs at $1.52 \mu \mathrm{m}$," IEEE Photon. Technol. Lett., vol. 10, no. 10, pp. 1419-1421, Oct. 1998.

[11] H. Ju, S. Zhang, H. de Waardt, G. D. Khoe, and H. J. S. Dorren, "SOAbased all-optical switch with subpicosecond full recovery," Opt. Exp., vol. 13, no. 3, pp. 942-947, Jan. 2004.

[12] H. Soto, D. Erasme, and G. Guekos, "Cross-polarization modulation in semiconductor optical amplifiers," IEEE Photon. Technol. Lett., vol. 11, no. 8, pp. 970-972, Aug. 1999.

[13] Y. Liu, M. T. Hill, D. Lenstra, H. de Waardt, G. D. Khoe, and H. J. S. Dorren, "Three-state all-optical memory based on three-coupled polarization switches," in Proc. ECOC, 2003, pp. 1-2. 
[14] P. A. Snow, I. E. Day, I. H. White, R. V. Penty, H. K. Tsang, R. S. Grant, Z. Su, W. Sibbett, J. B. D. Soole, H. P. Leblanc, A. S. Gosdz, N. C. Andreadakas, and C. Caneau, "Demonstration of polarization rotation gate in GaAs/AlGaAs multiquantum well waveguides," Electron. Lett., vol. 28, pp. 2346-2348, 1992.

[15] C.-C. Yang, "Intensity dependent polarization in a semiconductor multiple quantum-well amplifier," IEEE J. Quantum Electron., vol. 39, no. 4, pp. 1091-1097, Apr. 1993.

[16] N. Calabretta, Y. Liu, F. M. Huijskens, M. T. Hill, H. de Waardt, G. D. Khoe, and H. J. S. Dorren, "Optical signal processing based on selfinduced polarization rotation in a semiconductor optical amplifier," $J$. Lightw. Technol., vol. 22, no. 2, pp. 372-381, Feb. 2004.

[17] X. Yang, Z. Li, E. Tangdiongga, D. Lenstra, G. D. Khoe, and H. J. S. Dorren, "Sub-picosecond pulse generation employing an SOA based nonlinear polarization switch in a ring cavity," Opt. Express, vol. 12, pp. 2448-2453, 2004

[18] C. C. Yang, C. W. Lay, M. S. Lin, and D. W. Huang, "All-optical switching and passive mode-locking based on nonlinear polarization rotation in a semiconductor quantum well amplifier," Opt. Quantum Electron., vol. 28, pp. 1217-1227, 1996.

[19] W. Wang, K. Allaart, and D. Lenstra, "Semiconductor optical amplifier gain anisotropy: Confinement factor versus material gain," Electron. Lett., vol. 40, no. 5, pp. 1602-1603, Dec. 2004.

[20] C. Brosseau, Fundamentals of Polarized Light- A Statistical Approach. New York: Wiley, 1998, pp. 76-88.

[21] G. P. Agrawal, Nonlinear Fiber Optics, 3rd ed. San Diego, CA: Academic, 2001.

[22] S. A. Akhmanov, V. A. Vyslouhk, and A. S. Chirkin, Optics of Femtosecond Laser Pulses. New York: Amer. Inst. Phys., 1992, pp. 15-17.

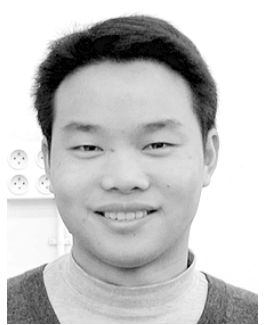

Z. Li was born in Sichuan, China, in 1979. He received the B.Sc. degree in optoelectronics and the M.Sc. degree in optics engineering from the University of Electronic Science and Technology of China, Chengdu, China, in 2000 and 2003, respectively.

$\mathrm{He}$ is currently working toward the Ph.D. degree at Eindhoven University of Technology, Eindhoven, The Netherlands, where he is involved in research on all-optical signal processing by employing semiconductor optical amplifier nonlinearities.

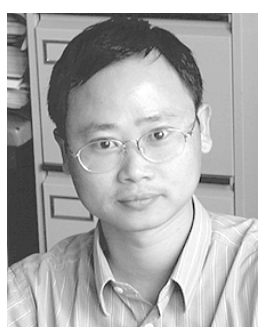

X. Yang received the B.S. degree in semiconductor physics from Jiangxi University, Nanchang, China, and the M.S. and Ph.D. degrees from the Department of Physics, Shanghai Jiao Tong University, Shanghai, China, 1992 and 1995, respectively.

After completing the Ph.D. degreee, he was employed as Associate Professor at Shanghai Jiao Tong University. From September 1999 to September 2001, he joined the Laboratoire Stereo-Chemie de Molecules \& Interactions (STIM), Ecole Normale Superieure de Lyon, France, as a Postdoctoral Researcher sponsored by a National Scholarship from French Ministry of Education. His research fields were organic nonlinear optical materials and devices. From October 2001 to November 2004, he was employed by the Department of Electrical Engineering, Eindhoven University of Technology, Eindhoven, The Netherlands. His interests mainly focused on ultrafast all-optical switching devices employing semiconductor optical amplifiers in optical telecommunication system. Since December 2004, he has been employed by Photonics System Group, University College Cork, Cork, Ireland, as Senior Postdoctoral Scientist. He is currently involved in all-optical signal processing in telecommunication system.

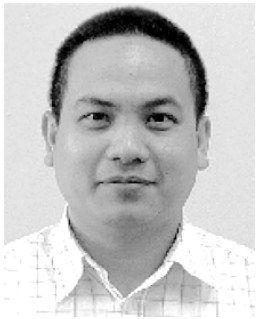

E. Tangdiongga was born in Makassar, Indonesia, in November 1968. He received the M.Sc. degree in electrical engineering and the Ph.D. degree from the Eindhoven University of Technology, Eindhoven, The Netherlands, in 1994 and 2001, respectively.

$\mathrm{He}$ joined the COBRA Research Institute, Eindhove University of Technology, in 1994 for conducting an experimental research on the topic of crosstalk in multiwavelength optical cross-connects.

He has been involved in the ACTS projects AC065 BLISS and AC332 APEX. During 2001-2003, he participated in the IST project FASHION for realization of $160 \mathrm{Gbit} / \mathrm{s}$ all-optical add-drop switching. He is currently working as a Senior Scientist in the same institute, responsible for realization of subpicosecond optical pulses and ultrafast all-optical demultiplexing for terabit per second data rates. His research interest covers crosstalk in WDM/OTDM networks, random signals, and nonlinearities in semiconductor-based optical devices.

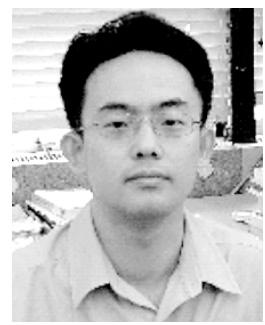

H. Ju was born in Seoul, Korea, in 1970. He received the B.S. degree in physics and the M.Sc. degree in quantum field theory of elementary particle physics at the Korea University, Seoul, Korea, in 1993 and 1997, respectively. He received the Ph.D. degree in condensed matter physics at the University of Oxford, Oxford, U.K., in 2003, before joining the ElectroOptical Communication Group, Eindhoven University of Technology, Eindhoven, The Netherlands as a Postdoctoral Researcher.

His research interests include ultrafast all-optical switching and memories by using nonlinear ultrafast carrier dynamics in semiconductor optical amplifiers.

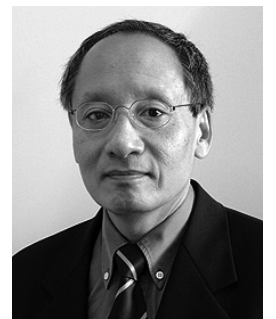

G.-D. Khoe (M'71-SM'85-F'91) was born in Magelang, Indonesia, on July 22, 1946. He received the Elektrotechnisch Ingenieur degree (cum laude) from the Eindhoven University of Technology, Eindhoven, The Netherlands, in 1971.

He decided to start research at the Dutch Foundation for Fundamental Research on Matter (FOM) Laboratory on Plasma Physics, Rijnhuizen, The Netherlands. In 1973, he moved to the Philips Research Laboratories to start research in the area of optical fiber communication systems. In 1983, he was appointed as part-time Professor at Eindhoven University of Technology. He became a Full Professor in 1994 and is currently Chairman of the Department of Telecommunication Technology and Electromagnetics (TTE). Most of his work has been devoted to single-mode fiber systems and components. Currently, his research programs are centered on ultrafast all-optical signal processing, high capacity transport systems, and systems in the environment of the users. He has more than 40 U.S. Patents and has authored and co-authored more than 100 papers, invited papers, and chapters in books. His professional activities include many conferences, where he has served in technical committees, management committees and advisory committees as a member or chairman. He is one of the founders of the Dutch COBRA University Research Institute and one of the three recipients of the prestigious "Top Research Institute Photonics" grant that is awarded to COBRA in 1998 by The Netherlands Ministry of Education, Culture and Science. In 2001, he brought four groups together to start a new international alliance called the European Institute on Telecommunication Technologies (eiTT).

Dr. Khoe was General Co-Chair of the ECOC 2001. He has been involved in journal activities, as Associate Editor, as a Member of the Advisory Board, or as Reviewer. Currently, he is an Associate Editor of IEEE JOURNAL OF QUANTUM ELECTRONICS. In Europe, he is closely involved in research programs of the European Community and in Dutch national research programs, as Participant, Evaluator, Auditor, and Program Committee Member. He has served in the IEEE/Lasers and Electro-Optics Society (LEOS) organization as European Representative in the Board of Governors (BoG), VP Finance and Administration, BoG Elected Member, and Member of the Executive Committee of the IEEE Benelux Section. He was founder of the LEOS Benelux Chapter He received the MOC/GRIN award in 1997. In 2004, he was the Junior Past President of LEOS. 


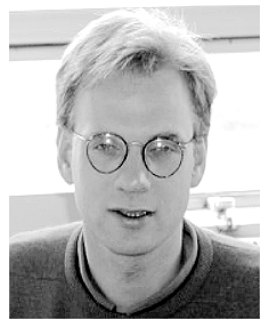

H. J. S. Dorren received the M.Sc. degree in theoretical physics and the Ph.D. degree from Utrecht University, Utrecht, The Netherlands, im 1991 and 1995, respectively.

After postdoctoral positions, he joined Eindhoven University of Technology, Eindhoven, The Netherlands, in 1996, where he presently serves as an Associate Professor. In 2002, he was also a Visiting Researcher at the National Institute of Industrial Science and Technology (AIST), Tsukuba, Japan. His research interests include optical packet switching, digital optical signal processing and ultrafast photonics.

Dr. Dorren currently serves as an Associate Editor for the IEEE JOURNAL OF QUANTUM ELECTRONICS.

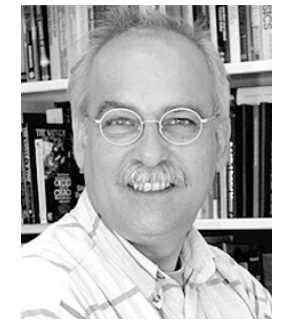

D. Lenstra was born in Amsterdam, The Netherlands, in 1947. He received the M.Sc. degree in theoretical physics from the University of Groningen, Groningen, The Netherlands, and the Ph.D. degree from Delft University of Technology, Delft, The Netherlands. His thesis work was on polarization effects in gas lasers.

Since 1979, he researched topics in quantum electronics, laser physics, and condensed matter physics. In 1991, he joined the Vrije Universiteit, Amsterdam, The Netherlands, holding a Chair in Theoretical Quantum Electronics. In 2000, he became also part-time Professor in the Department of Electrical Engineering, Eindhoven University of Technology, Eindhoven, The Netherlands, where he presently occupies a Chair on Ultrafast Photonics as part-time Professor. His research themes are nonlinear and ultrafast dynamics of semiconductor optical amplifiers and diode lasers, quantum optics in small semiconductor structures, and near-field optics. He has authored and coauthored more than 200 publications in international scientific journals and edited or coedited six books and several special journal issues.

Dr. Lenstra is a member of IEEE Lasers and Electro-Optics Society (LEOS), OSA, and EPS, and acts regularly as Program Chair for international meetings. 